\title{
EUS-guided 22-gauge fine needle biopsy versus single-incision with needle knife for the diagnosis of upper gastrointestinal subepithelial lesions: a randomized controlled trial
}

\section{(ㄷ)(우우}

Authors

Omid Sanaei ${ }^{*}, 1$, Glòria Fernández-Esparrach ${ }^{*}$,2,3,4, Carlos De La Serna-Higuera ${ }^{5}$, Silvia Carrara6, Vivek Kumbhari', Mohamad H. El Zein ${ }^{1}$, Amr Ismail' ${ }^{1}$, Angels Ginès ${ }^{2,3,4}$, Oriol Sendino ${ }^{2,3,4}$, Andrea Montenegro ${ }^{2}$, Alessandro Repici', Daoud Rahal7, Olaya I. Brewer Gutierrez¹, Robert Moran'1, Juliana Yang1', Nasim Parsa', Christopher Paiji', Mohamad Aghaie Meybodi ${ }^{1}$, Eun Ji Shin ${ }^{1}$, Anne Marie Lennon ${ }^{1}$, Anthony N. Kalloo ${ }^{1}$, Vikesh K. Singh ${ }^{1}$, Marcia Irene Canto ${ }^{1}$, Mouen A. Khashab ${ }^{1}$

Institutions

1 Division of Gastroenterology and Hepatology, Johns Hopkins Medical Institution, Baltimore, Maryland, United States

2 Endoscopy Unit, Gastroenterology Department, ICMDiM, Hospital Clinic, University of Barcelona, Catalonia, Spain

3 Institut d'Investigacions Biomèdiques August Pi i Sunyer (IDIBAPS), Barcelona, Spain

4 Centro de Investigación Biomédica en Red de Enfermedades Hepáticas y Digestivas (CIBEREHD), Barcelona, Spain

5 Gastroenterology Department, Hospital Universitario Rio Hortega, Valladolid, Spain

6 Digestive Endoscopy Unit, Division of Gastroenterology, Humanitas Clinical and Research Center - IRCCS, Rozzano, Italy

7 Department of Pathology, Humanitas Clinical and Research Center, Rozzano, Italy

submitted 6.9.2019

accepted after revision 18.11.2019

Bibliography

DOI https://doi.org/10.1055/a-1075-1900 |

Endoscopy International Open 2020; 08: E266-E273

(c) Georg Thieme Verlag KG Stuttgart · New York

elSSN 2196-9736

Corresponding author

Mouen A. Khashab, MD, Johns Hopkins Hospital, 1800

Orleans Street, Sheikh Zayed Tower, Baltimore, MD 21287

Fax: +1-443-683-8335

mkhasha1@jhmi.edu

\section{ABSTRACT}

Background and study aims EUS-FNA has suboptimal accuracy in diagnosing gastrointestinal subepithelial tumors (SETs). EUS-guided 22-gauge fine needle biopsy (EUS-FNB) and single-incision with needle knife (SINK) were proposed to increase accuracy of diagnosis. This study aimed to prospectively compare the diagnostic accuracy and safety of EUS-FNB with SINK in patients with upper gastrointestinal SETs.

Patients and methods All adult patients referred for EUS evaluation of upper gastrointestinal SETs $\geq 15 \mathrm{~mm}$ in size were eligible for inclusion. Patients were randomized to undergo EUS-FNB or SINK. Lesions were sampled with a 22gauge reverse beveled core needle in the EUS-FNB group and by a conventional needle-knife sphincterotome and biopsy forceps in the SINK group. Patients were blinded to the technique used. The primary outcome was diagnostic accuracy. Secondary outcomes included adverse events, histological yield and procedure duration. Study enrollment was terminated early due to poor recruitment.

Results A total of 56 patients (31 male ( $55.37 \%$ ); mean age, $67.41 \pm 12.70$ years) were randomized to either EUSFNB $(n=26)$ or SINK $(n=30)$. Technical success was $96.15 \%$ and $96.66 \%$, respectively. The majority of lesions were gastrointestinal stromal tumors $(51.78 \%)$. No significant difference was found between EUS-FNB and SINK in terms of diagnostic accuracy for a malignant or benign disease (76\% vs. $89.28 \%$, respectively; $P=0.278$ ). The rate of adverse events (none severe) was also comparable (7.69\% vs. $10 \%$, respectively; $P=1.0$ ) including two abdominal pain episodes in the EUS-FNB group compared to two delayed bleeding (one requiring hospitalization and radiologic embolization) and 1 abdominal pain in the SINK group.

Conclusion EUS-FNB and SINK are equally effective techniques for upper gastrointestinal SETs sampling. SINK can be associated with mild to moderate delayed bleeding.

\footnotetext{
* Drs. Sanaei and Fernández-Esparrach contributed equally.
} 


\section{Introduction}

Upper gastrointestinal subepithelial tumors (SETs) are incidentally found in $0.8 \%$ to $2 \%$ of patients undergoing upper endoscopy [1]. These include lipomas, leiomyomas, aberrant pancreas (pancreas rest), varices, carcinoids, gastrointestinal stromal tumors (GISTs), and lymphomas [1,2]. Various management options exist depending on the pathologic diagnosis and include surveillance, endoscopic or surgical removal or, in selected cases of GISTs, targeted therapy with tyrosine kinase inhibitors [3]. Therefore, a correct diagnosis is important to guide subsequent management, especially as cross-sectional imaging has low accuracy. EUS-guided sampling has been the technique of choice for tissue procurement from SETs of the digestive tract [1].

EUS-guided fine-needle aspiration (EUS-FNA) is the usual method of sampling SETs; however, its diagnostic yield is low (about $60 \%$ ) and immunohistochemical analysis is only possible in approximately $75 \%$ of cases $[4,5]$. Thus, EUS-guided fineneedle biopsy (EUS-FNB) has been developed in recent years for the purpose of obtaining core samples that allow for histological assessment. However, the overall reported diagnostic yield of EUS-FNB is still modest (75\% to $86 \%$ ) [5, 6-9].

In recent years, the single-incision with needle knife (SINK) technique has been developed to overcome suboptimal results of EUS-guided sampling techniques in SETs. This technique involves initial exposure of the SET with needle knife mucosal incision followed by direct tumor sampling with a forceps [10]. To date, three retrospective studies have evaluated the efficacy of SINK. De la Serna et al. introduced the SINK technique in a retrospective series of 14 cases and reported a diagnostic yield of $93 \%$ [10]. In two other retrospective studies, including 31 and 49 patients, the diagnostic yield was $87 \%$ and $91 \%$, respectively $[11,12]$. Performance of the SINK technique with EUS-FNB is currently unknown. The aim of this study was to prospectively compare the efficacy and safety of EUS-FNB with SINK in patients with upper gastrointestinal SETs.

\section{Patients and methods}

This is a single-blinded randomized clinical trial (NCT02282111) conducted at four tertiary referral hospitals (three in Europe and one in the United States). The study was approved by the institutional review board at all participating centers. Eligible participants were adult patients who were referred for EUS evaluation of upper gastrointestinal SETs that are at least $15 \mathrm{~mm}$ in diameter. Of note, 10 months after initiation of the study, the minimum lesion diameter for inclusion was decreased from $20 \mathrm{~mm}$ to $15 \mathrm{~mm}$ due to suboptimal recruitment. Exclusion criteria were endoscopically non-bulging lesions, upper gastrointestinal SETs $<15 \mathrm{~mm}$ in size as measured by EUS, lesions not necessitating tissue acquisition (i.e. lipomas, varices), cystic lesions, uncorrectable coagulopathy (international normalized ratio (INR) $>1.5$ or platelets $<50,000 / \mu \mathrm{L})$, patients with stigmata of portal hypertension, patients with post-surgical upper gastrointestinal anatomy
(Roux-en-Y gastric bypass, esophagectomy, etc.), pregnant women and refusal to consent.

\section{Randomization and enrollment}

Using a computer-generated list, randomization was done in blocks of four and six. An investigator at the coordinating center, without clinical involvement in the study, prepared and distributed randomization envelopes for all participating centers. Patients were randomly allocated with a 1:1 ratio to receive SINK or EUS-FNB. Written informed consent was obtained from all the participants before enrollment in the study. All data were collected using the same case report form.

\section{EUS-FNB technique}

Linear EUS was used with color and pulsed Doppler to scan the area for vessels. The lesion was then sampled with a 22-gauge reverse beveled needle (ProCore, Cook Medical Inc., WinstonSalem, NC) using the slow capillary suction and fanning techniques with five to 15 to-and-fro movements with each pass. A total of four passes were performed and then the procedure terminated. No cytopathologist was present to review the specimens. If the samples were of insufficient quality as per the impression of the endoscopist (defined as obtaining only tiny fragments, no visible core tissue or it appeared that only blood clot was present), an alternative method (e.g. EUS-FNA, SINK, etc) was performed at the discretion of the endoscopist.

\section{SINK technique}

A conventional needle-knife sphincterotome (Microknife $\mathrm{XL}$; Boston Scientific Inc, Natick, Massachusetts, United States) connected to an electrosurgical unit (ERBE, Tübingen, Germany) was utilized. The setting used was ENDOCUT I, Effect 3, Duration 2 and Interval 1. Under direct endoscopic vision, a 6to $12-\mathrm{mm}$ linear incision was made from the periphery of the lesion to its highest convexity zone. Care was taken to make the incision deep enough such that it penetrated the mucosa and submucosa. A conventional biopsy forceps (Radial Jaw4, Boston Scientific, Natick, Massachusetts, United States) was then introduced through the incision, and two bites were obtained per pass. A total of four passes were performed by passing the biopsy forceps through the incision on each occasion. In case of technical failure, the endoscopist was permitted to choose another method to obtain biopsies (e.g. EUS-FNB, EUSFNA, etc.). The mucosal incision was then closed with endoclips whenever possible ( $\triangleright$ Fig. 1) [10].

\section{Pathologic evaluation}

Specimens were placed in buffered formalin and processed as normal forceps biopsy specimens with special care taken not to lose small specimen fragments. Pathologists did not have any knowledge of which techniques were used. All other clinical history and endoscopic information were provided. The specimens were subjected to immunostaining at the pathologist's discretion. 

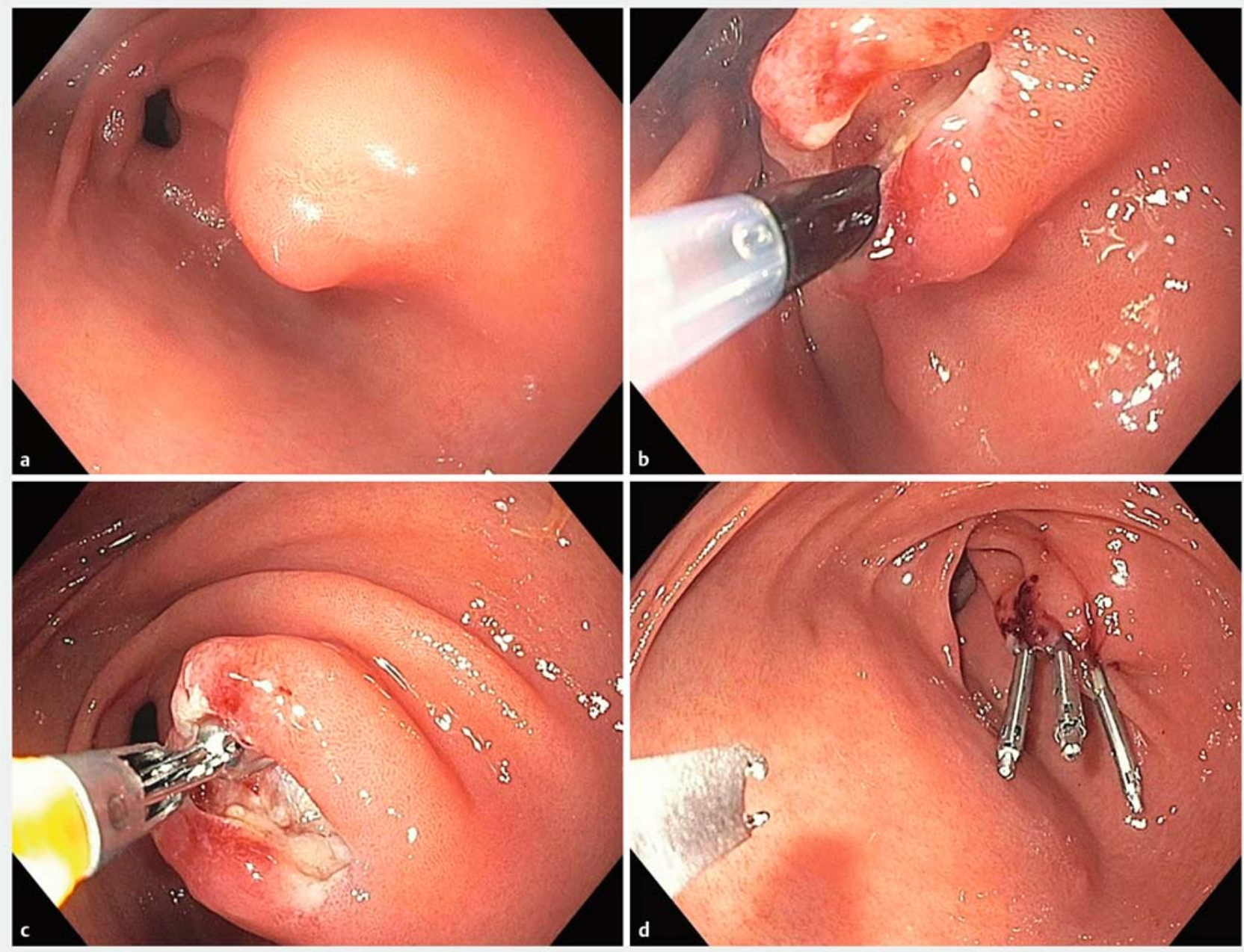

- Fig. 1 Single incision needle knife (SINK) technique. a Endoscopic image showing a 15-mm subepithelial lesion in the antrum, $\mathbf{b}$ A mucosal incision was made using a needle knife exposing the subepithelial tumor. $\mathbf{c}$ The tumor was then directly sampled with a biopsy forceps. $\mathbf{d}$ The incision was closed using hemoclips.

\section{Follow-up}

Patients were observed for immediate complications in the recovery area for 1 to 2 hours and were discharged on the same day. Telephone contact was made the day after the procedure as well as 1 week later to monitor for any delayed adverse events (AEs). Long-term clinical follow-up was determined by the pathological findings.

\section{Final diagnosis and definition}

In the absence of surgical resection, histologic diagnosis made by either technique was considered the gold standard. When diagnosis could not be made based on the histology, a definitive diagnosis was established on the basis of long-term follow-up, surgery, or repeat biopsy. A positive diagnosis of a specific malignancy or of a specific benign disease by either technique was accepted as a true positive. A histological diagnosis of atypical cells or abnormal cells was considered as a negative result. For malignancy, an "accurate diagnosis" was considered one in which a tissue diagnosis obtained was compati- ble with that seen by subsequent surgery, alternative biopsy method, or clinical follow up. The diagnostic accuracy was defined as the ratio between the sum of true positive and true negative values divided by the total number of cases with final diagnosis.

\section{Outcomes}

The primary outcome was to compare the diagnostic accuracy of EUS-FNB with SINK. The secondary outcomes were to compare histological yield, technical failure (inability to complete the four assigned passes as determined by randomization), AEs with their severity being rated according to ASGE lexicon [13], procedure duration (time from the beginning of the incision or needle insertion, to completion of tissue acquisition per protocol) and contribution of immunohistochemistry (when needed) between the two techniques. 


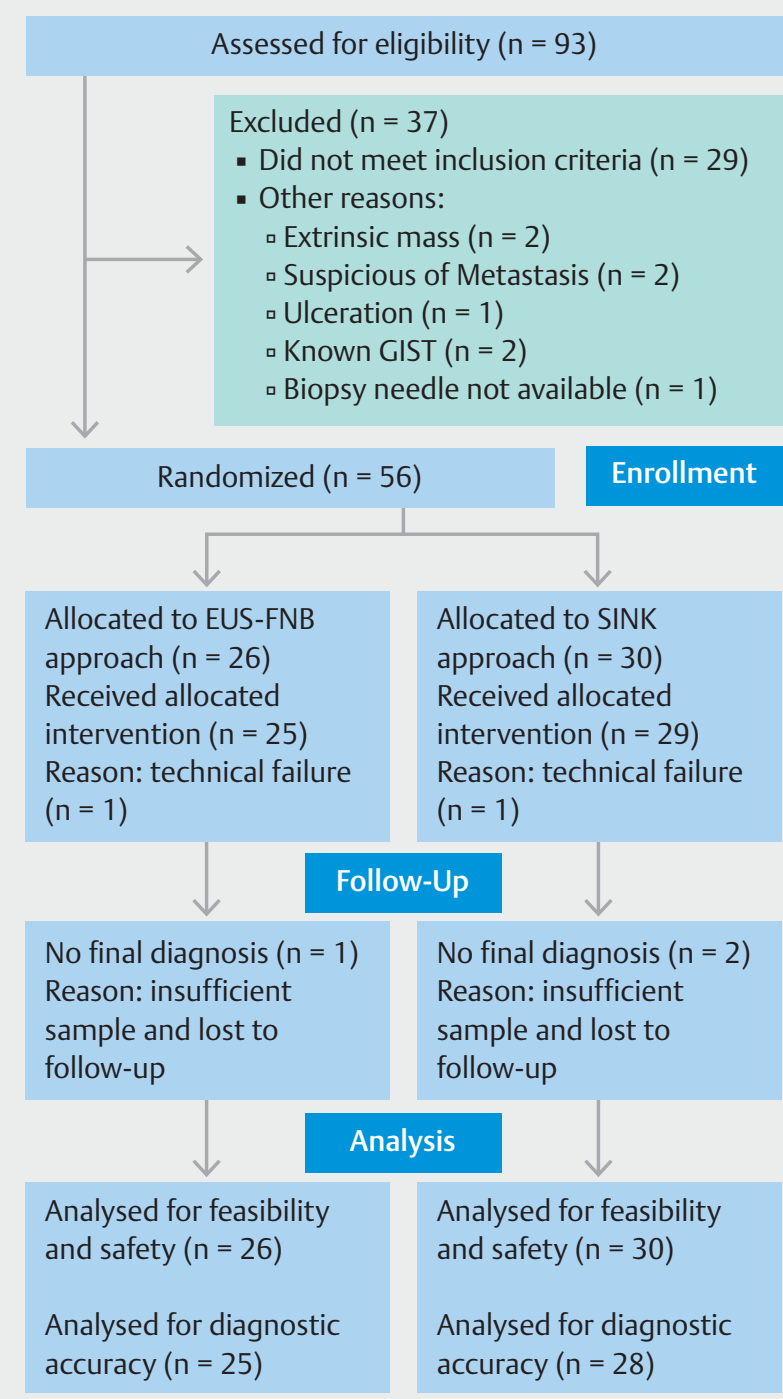

Fig. 2 EUS-FNB vs SINK flow diagram.

\section{Study statistics}

The hypothesis of the study was that the diagnostic accuracy of SINK technique is "superior" in obtaining histological samples as compared to EUS-FNB technique. With the assumption that the diagnostic yield of EUS-FNB be 70\% [5] and that of SINK be $95 \%$ [10] and considering $\alpha=5 \%$ and $1-\beta=80 \%$, the required sample size was calculated as 36 patients in each group. Having considered a $20 \%$ drop-out rate, a total of 90 patients, 45 in each arm was calculated. It should be noted that the $70 \%$ assumed diagnostic yield was selected based on publications using the Trucut needle [5]. The reason was that at the time of the study design in 2013, there were no studies using FNB. The study was terminated after randomization of 56 patients. In fact, the interim data analysis demonstrated that based on the accuracy in both groups, 131 patients in each group would need to be recruited to achieve differences with statistical power to be as high as $80 \%$. Such a potentially high number of patients together with the slow recruitment made the continua- tion of the study not feasible and we decided to close the inclusion.

The study results are reported as mean and standard deviation (SD) or median and interquartile range (IQR) for quantitative variables and percentages for categorical variables. Continuous variables including age, greatest diameter of the lesion and procedure duration were compared by Student's t-test or Mann-Whitney U test where appropriate. Categorical variables were compared using chi-square test or Fisher's exact test. All statistical analyses were performed using SPSS software (version 17.0; SPSS, Chicago, Illinois, United States), with results considered significant at $P<0.05$.

\section{Results}

A total of 93 patients were assessed for eligibility between September 2014 and July 2017, of whom 56 were randomly assigned to EUS-FNB $(n=26)$ or SINK $(n=30)(\vee$ Fig. 2$)$. The mean age was $67.41 \pm 12.7$ years with a slight male preponderance (n $=31,55.35 \%$ ). There was no statistically significant difference between the two groups in terms of baseline characteristics ( $\triangleright$ Table 1$)$. The majority of lesions in both groups were GISTs (17 $(65.38 \%)$ in the EUS-FNB group versus $12(40 \%)$ in the SINK group; $P=0.06)(\vee$ Table 2$)$. The index procedure was the most frequent diagnostic method used to establish the final diagnosis.

EUS-FNB and SINK were successfully performed in $96.15 \%$ (95\% Cl, 81.10\%-99.31\%) and $96.66 \%(95 \% \mathrm{Cl}, 83.32 \%-$ $99.40 \%)$ of cases, respectively $(P=1.0)$. Technical success and other major outcomes of the study are described in $>$ Table 3 . In one patient in the EUS-FNB group, the procedure was aborted before obtaining biopsy due to difficulty in advancing the needle through the lesion which was in the stomach. The EUSFNA was then used as an alternative approach (2 passes using 22-gauge EchoTip needle) which was not successful in yielding a histopathological diagnosis. Finally, surgical pathology confirmed the diagnosis of schwannoma. Similarly, one SINK procedure failed due to intraprocedural bleeding. In this patient, the diagnosis of GIST was confirmed by surgical pathology.

In the SINK group, the mean \pm SD length of incision was 8.53 $\pm 1.75 \mathrm{~mm}$. In 22/30 (73.33\%) patients, endoclips were successfully used for incision closure with the median (IQR) number of $2(1-2)$ clips. The median (IQR) procedure duration was 12 minutes (range 8-20) in the EUS-FNB as compared to 11 minutes (range 8-17) in the SINK group ( $P=0.79$ ).

The rate of AEs was $7.69 \%(95 \% \mathrm{Cl}, 2.13 \%-24.14 \%)$ in the EUS-FNB group as compared to $10 \%(95 \% \mathrm{Cl}, 3.45 \%-25.62 \%)$ in the SINK group $(P=1.0)$. Specifically, there was one patient with mild and one with moderate abdominal pain in the EUSFNB group which were managed conservatively. In the SINK group there was one patient with mild abdominal pain that was managed conservatively and two delayed bleeding episodes occurring 6 days after the procedure. One of the bleeding episodes presented as a self-limited melena in a patient with a plexiform angiomyxoid myofibroblastic tumor and was rated as mild; the other one was rated as moderate occurring in a patient with a $23 \times 24 \mathrm{~mm}$ GIST ultimately requiring arterial 
- Table 1 Baseline characteristics.

\begin{tabular}{|c|c|c|c|c|}
\hline & & EUS-FNB $(n=26)$ & SINK $(n=30)$ & $P$ value \\
\hline \multicolumn{2}{|l|}{ Age, mean $\pm S D$, years } & $68.7 \pm 11.9$ & $66.3 \pm 13.4$ & 0.47 \\
\hline Gender, n (\%) & Male & $15(57.7)$ & $16(53.3)$ & 0.74 \\
\hline \multirow[t]{3}{*}{ Layer of origin, n (\%) } & Submucosa & $7(26.9)$ & $9(30)$ & \multirow[t]{3}{*}{0.72} \\
\hline & Muscularis propria & $17(65.4)$ & $17(56.7)$ & \\
\hline & Indeterminate & $2(7.7)$ & $413.3)$ & \\
\hline \multicolumn{2}{|c|}{ Greatest diameter, median (IQR), mm } & $23.5(20-39)$ & $25(20-30)$ & 0.75 \\
\hline \multirow[t]{4}{*}{ Echogenicity, n (\%) } & Hypoechoic & $19(73.1)$ & $22(73.3)$ & \multirow[t]{4}{*}{0.73} \\
\hline & Isoechoic & $5(19.2)$ & $7(23.3)$ & \\
\hline & Hyperechoic & $1(3.8)$ & $1(3.3)$ & \\
\hline & Mixed echogenicity & $1(3.8)$ & 0 & \\
\hline Calcification, n (\%) & Yes & 0 & $4(13.3)$ & 0.11 \\
\hline Cystic component, n (\%) & Yes & $7(26.9)$ & $3(10)$ & 0.16 \\
\hline \multirow[t]{5}{*}{ Location of the lesion, $n$ (\%) } & Esophagus & $1(3.8)$ & $1(3.3)$ & \multirow[t]{5}{*}{0.89} \\
\hline & Gastric fundus & $7(26.9)$ & $9(30)$ & \\
\hline & Gastric body & $12(46.2)$ & $12(40)$ & \\
\hline & Antrum & $4(15.4)$ & $7(23.3)$ & \\
\hline & Duodenum & $2(7.7)$ & $1(3.3)$ & \\
\hline Anticoagulation use, n (\%) & Yes & $2(7.7)$ & $7(23.3)$ & 0.15 \\
\hline
\end{tabular}

- Table 2 Final diagnosis of lesions.

\begin{tabular}{|c|c|c|}
\hline & $\begin{array}{l}\text { EUS-FNB } \\
(n=26)\end{array}$ & SINK $(n=30)$ \\
\hline GIST, n (\%) & $17(65.8)$ & $12(40)$ \\
\hline Fibroma, n (\%) & 0 & $1(3.3)$ \\
\hline Mesenchymal tumor, n (\%) & $1(3.8)$ & 0 \\
\hline Chemical gastritis, n (\%) & 0 & $2(6.6)$ \\
\hline Leiomyoma, n (\%) & $2(7.7)$ & $5(16.7)$ \\
\hline Lipoma, n (\%) & $1(3.8)$ & $4(13.3)$ \\
\hline Inflammatory fibroid polyp, n (\%) & 0 & $1(3.3)$ \\
\hline Heterotopic pancreas, n (\%) & $1(3.8)$ & 0 \\
\hline Intragastric ectopic spleen, n (\%) & 0 & $1(3.3)$ \\
\hline Schwannoma, n (\%) & $1(3.8)$ & $1(3.3)$ \\
\hline Neuroendocrine tumor, n (\%) & $2(7.7)$ & 0 \\
\hline $\begin{array}{l}\text { Plexiform angiomyxoid } \\
\text { myofibroblastic tumor, n (\%) }\end{array}$ & 0 & $1(3.3)$ \\
\hline Unavailable ${ }^{1}, \mathrm{n}(\%)$ & $1(3.8)$ & $2(6.6)$ \\
\hline $\begin{array}{l}\text { GIST, Gastrointestinal stromal tumor } \\
{ }^{1} \text { Histological diagnosis not reached w } \\
\text { up }\end{array}$ & JS-FNB or S & st to follow- \\
\hline
\end{tabular}

embolization. In the latter patient, the index procedure had been complicated with immediate bleeding controlled by endoclips and a detachable snare.

Final diagnosis was made by the index procedure and surgery in 34 patients $(60.71 \%$ ) and 14 patients ( $25 \%$ ), respectively. In particular, the index procedure was the final diagnostic method in 11 patients (42.15\%) and 23 patients (76.66\%) in the EUS-FNB and SINK groups, respectively. In the same way, surgical pathology was the method of final diagnosis in 10 patients (38.46\%) and four patients (13.33\%), respectively. Apart from three patients ( $5.35 \%$ ) with inadequate sample who were lost to follow-up with unavailable final diagnosis, the remaining seven patients (12.5\%) were diagnosed either by surgical pathology, clinical follow-up at 6 to 12 months or alternative diagnostic methods including EUS-FNA done at the time of procedure in one patient as well as repeat SINK done 6 weeks later at a separate session in two patients. The EUS-FNA led to the diagnosis of leiomyoma and repeat SINK diagnosed one GIST and one heterotopic pancreas.

In the calculation of diagnostic accuracy, we excluded one patient in the EUS-FNB group and two patients in the SINK group who were lost to follow-up and did not have a final diagnosis ( $\triangleright$ Fig.2). There was no significant difference between the two groups in terms of diagnostic accuracy for malignant or benign disease (19/25 [76\%; $95 \% \mathrm{Cl}, 59.25 \%-92.74 \%]$ for EUS-FNB as compared with 25/28 [89.28\%; $95 \% \mathrm{Cl}, 77.82 \%-$ 
- Table 3 Major study outcomes.

\begin{tabular}{|c|c|c|c|}
\hline & EUS-FNB $(n=26)$ & SINK $(n=30)$ & $P$ value \\
\hline Technical success, n (\%) & $25(96.15)$ & $29(96.66)$ & 1.0 \\
\hline Procedure duration, median (IQR), min & $12(8-20)$ & $11(8-17)$ & 0.79 \\
\hline Adequate sample at endoscopist discretion, n (\%) & $23(88.46)$ & $29(96.66)$ & 0.32 \\
\hline Adequate sample at pathologist discretion, $\mathrm{n}(\%)$ & $20(76.92)$ & $26(86.66)$ & 0.5 \\
\hline Possible IHC evaluation, $\mathrm{n}(\%)^{1}$ & $13 / 18(72.22)$ & $18 / 19(94.73)$ & 0.09 \\
\hline Diagnostic accuracy, $\mathrm{n}(\%)^{2}$ & $19 / 25(76 \%)$ & $25 / 28(89.28)$ & 0.28 \\
\hline \multicolumn{4}{|c|}{$\begin{array}{l}\text { IHC, immunohistochemistry; IQR, interquartile range } \\
1 \text { IHC was requested in } 18 \text { patients in the EUS-FNB group and } 19 \text { patients in the SINK group. } \\
{ }^{2} \text { Calculation was done after exclusion of one patient in the EUS-FNB group and two patients in the SINK group who were lost to follow-up and did not have a final } \\
\text { diagnosis. }\end{array}$} \\
\hline
\end{tabular}

$100 \%$ ] for SINK; $P=0.278)$. We achieved similar results after removal of patients with the diagnosis of lipoma according to the study's drop out criteria (18/24 [75\%; $95 \%$ Cl, 57.67\%-92.32\%] for EUS-FNB as compared with 21/24 [87.5\%; $95 \% \mathrm{CI}, 74.26 \%$ $100 \%$ ] for SINK; $P=0.461$ ).

\section{Discussion}

Gastrointestinal SETs constitute a subset of lesions in which a histological diagnosis with immunohistochemistry is deemed necessary for the diagnosis. Although EUS-FNA has a high accuracy in most clinical settings [14-16], its accuracy is lower in this type of lesions $[4,5]$. EUS-FNB provides a core tissue biopsy which could theoretically be more useful than EUS-FNA in patients with SETs. Studies evaluating the use of EUS-FNB, have reported an overall accuracy ranging from $74 \%$ to $86 \%$ for this technique $[4,7-9]$; results that are not very different than those obtained using EUS-FNA. Of note, in a meta-analysis of 17 studies including 978 procedures [4], the diagnostic yield of EUS-guided sampling for upper gastrointestinal subepithelial lesions (SELs) was reported as $60 \%(95 \% \mathrm{Cl}, 55 \%-65 \%)$. This study concluded that EUS-guided needle sampling is only a moderately effective method for pathological diagnosis of upper gastrointestinal SELs and neither the procedure type (FNA or FNB) nor the needle size affected the overall diagnostic rate. However, a very recent study suggests a potential role for EUS-FNB for the acquisition of adequate samples for IHC analysis [17].

There are only three studies evaluating performance of SINK [10-12]. All of them were retrospective studies with between 14 to 49 patients. One study reported SINK to have a diagnostic yield of $93 \%$ and found it superior to EUS-FNA in providing a final histological diagnosis ( $75 \%$ vs. $12.5 \%$; $P=0.23$ ) [10]. In the other two studies, however, the performance of SINK was reported as $89 \%$ diagnostic accuracy in one [11] and $86 \%$ tissue sufficiency to reach a definite pathologic diagnosis in the other [12]. Small, retrospective studies have inherent issues including being prone to biases and confounding factors. The aim of this study was to prospectively determine whether SINK is superior to EUS-FNB.
The current study is the first randomized trial specifically designed to compare efficacy and safety of EUS-FNB and SINK in patients with upper gastrointestinal SETs. We hypothesized that SINK was superior to EUS-FNB in diagnosis of upper gastrointestinal SETs. Although the diagnostic accuracy of SINK was numerically higher than that of EUS-FNB, the difference between the two rates did not reach statistical significance. Of note, the $76 \%$ diagnostic accuracy for EUS-FNB in our study was comparable to other studies reporting rates of $75 \%$ to $92 \%$ for EUS-FNB using similar 22-gauge ProCore needle $[6,18,19]$. In the same way, the $89.28 \%$ diagnostic accuracy of SINK was comparable to the rates of $87 \%$ to $93 \%$ reported in other studies [10-12].

When IHC was deemed necessary for diagnosis, in this study SINK was superior to EUS-FNB for obtaining tissue samples which were suitable for IHC. These results are similar to a study by Shimamura et al. in which $91 \%$ of samples obtained by SINK were suitable for immunohistochemical evaluation [12]. Of note, although the $22 \%$ difference was not statistically significant, from clinical standpoint the difference could be considered as a significant finding given the pivotal role of $\mathrm{IHC}$ in proper diagnosis of gastrointestinal SETs.

With respect to AEs, and in contrast to prior studies that reported a $0.4 \%$ risk of severe bleeding [20], we did not find any hemorrhagic AE following EUS-FNB. In addition, there were no episodes of sepsis or fever suggesting the use of prophylactic antibiotics when using a 22-gauge reverse beveled needle or doing SINK. However, in contrast to prior studies of SINK for SETs, there were two delayed bleeding AEs [10-12].

Several studies have investigated the accuracy of different EUS-guided tissue sampling combinations and demonstrated that adopting a combined rather than single-technique approach will improve the diagnostic accuracy [21-23]. With this in mind and given the fact that the rate of successful immunohistochemical analysis on adequate samples in our study was higher with SINK than with EUS-FNB, and that the adequacy can be easily assessed by the endosonographer, one possible strategy is to perform SINK initially followed by EUS-FNB only if it fails. Based on this, the next step would be comparison of either SINK or EUS-FNB alone with the combination of the two 
techniques. Finally, with the advent of newer Franseen and forktip needles with reported promising diagnostic yield, conducting randomized trials comparing SINK with EUS-FNB using these new needles is warranted [24, 25].

The major strength of this study is that the EUS-FNB and SINK were compared in a randomized fashion. Further, the multicenter nature of the study enhances the generalizability of the trial. The main limitation of this study is that we included fewer patients than the calculated sample size. As discussed above, the recruitment of 131 patient is each group seemed unfeasible given the slow recruitment rate. The second limitation is that the design of the study could have been detrimental for the EUS-FNB because of the following reasons: the design was not cross-over and the number of GIST was slightly higher in the EUS-FNB group ( $65.38 \%$ vs $40 \%$ ). This type of tumor is the most difficult to diagnose and for the SINK technique to be feasible, we only included bulging tumors measuring $15 \mathrm{~mm}$ or more. Given that EUS-FNB can be used for smaller non-bulging tumors, had we included lesions smaller than $15 \mathrm{~mm}$ in a crossover design, the results could have benefited EUS-FNB.

\section{Conclusion}

In conclusion, in this prospective, randomized, multicenter study, EUS-FNB and SINK have a similar overall performance for the diagnosis of gastrointestinal SETs although SINK can be associated with mild to moderate delayed bleeding. This study suggests EUS-FNB as first line technique for gastrointestinal SETs whereas SINK could represent a salvage technique after failed diagnosis with FNB for intraluminal SETs. Of note, proper qualification of the lesion by EUS is warranted when SINK is chosen to be done.

\section{Competing interests}

Dr. Khashab is a consultant and on medical advisory board for Boston Scientific and Olympus and a consultant for Medtronic. Dr. Carrara is a consultant, speaker and teacher for Olympus, recipient of Grant/Research support from Boston Scientific. Dr. Fernández-Esparrach is a consultant for CDx Diagnostics and teacher for Norgine Iberia and Olympus Spain. Dr. Kumbhari is a consultant for Boston Scientific, Apollo Endosurgery, Medtronic, Pentax Medical and ReShape Medical, and recipient of research support from ERBE and Apollo Endosurgery. Dr. Ginès is a consultant for Cook Medical. Dr. Repici is a consultant and on advisory board for Norgine and Lumendi, recipient of Grant/Research Support from Boston Scientific and Fujifilm. Dr. Shin is a consultant for Boston Scientific, Medtronic and C2 Therapeutics.Dr. Kalloo is a founding member, equity holder, and consultant for Apollo Endosurgery. Dr. Singh is a consultant for Abbvie, Ariel Precision Medicine, and Akcea Therapeutics. Dr. Canto is a recipient of Grant/Research Support from C2 Therapeutics.
References

[1] Dumonceau J-M, Deprez PH, Jenssen C et al. Indications, results, and Clinical impact of endoscopic ultrasound (EUS)-guided sampling in gastroenterology: European Society of Gastrointestinal Endoscopy (ESGE) Clinical Guideline - Updated January 2017. Endoscopy 2017; 49: 605-714

[2] Akahoshi KSY, Matsui N et al. Preoperative diagnosis of gastrointestinal stromal tumor by endoscopic ultrasound-guided fine needleaspiration. World J Gastroenterol 2007; 13: 2077-82

[3] Lai EC, Lau SH, Lau WY. Current management of gastrointestinal stromal tumors-a comprehensive review. Int J Surg 2012; 10: 334340

[4] Zhang X-C, Li Q-L, Yu Y-F et al. Diagnostic efficacy of endoscopic ultrasound-guided needle sampling for upper gastrointestinal subepithelial lesions: a meta-analysis. Surg Endosc 2016; 30: 2431-2441

[5] Fernández-Esparrach G, Sendino O, Solé M et al. Endoscopic ultrasound-guided fine-needle aspiration and trucut biopsy in the diagnosis of gastric stromal tumors: a randomized crossover study. Endoscopy 2010; 42: 292-299

[6] Schlag C, Menzel C, Götzberger M et al. Endoscopic ultrasound-guided tissue sampling of small subepithelial tumors of the upper gastrointestinal tract with a 22-gauge core biopsy needle. Endosc Int Open 2017; 5: E165-E171

[7] Lee M, Min BH, Lee $\mathrm{H}$ et al. Feasibility and diagnostic yield of endoscopic ultrasonography-guided fine needle biopsy with a new core biopsy needle device in patients with gastric subepithelial tumors. Medicine (Baltimore) 2015; 94: e1622

[8] Lee JH, Cho C], Park YS et al. EUS-guided 22-gauge fine needle biopsy for the diagnosis of gastric subepithelial tumors larger than $2 \mathrm{~cm}$. Scand J Gastroenterol 2016; 51: 486-493

[9] Han JP, Lee TH, Hong S] et al. EUS-guided FNA and FNB after on-site cytologic evaluation in gastric subepithelial tumors. J Dig Dis 2016; 17: $582-587$

[10] de la Serna-Higuera C, Pérez-Miranda M, Díez-Redondo P et al. EUSguided single-incision needle-knife biopsy: description and results of a new method for tissue sampling of subepithelial gastrointestinal tumors (with video). Gastrointest Endosc 2011; 74: 672-676

[11] Kim JS, Kim BW, Kim G] et al. Diagnostic yield of endoscopic ultrasonography-guided single-incision needle knife biopsy for gastric subepithelial tumors: comparison with resected specimens. Surg Endosc 2016; 30: 5304-5309

[12] Shimamura Y, Hwang J, Cirocco M et al. Efficacy of single-incision needle-knife biopsy for sampling subepithelial lesions. Endosc Int Open 2017; 5: E5-E10

[13] Cotton PB, Eisen GM, Aabakken L et al. A lexicon for endoscopic adverse events: report of an ASGE workshop. Gastrointest Endosc 2010; 71: 446-454

[14] Vazquez-Sequeiros E, Wiersema MJ, Clain JE et al. Impact of lymph node staging on therapy of esophageal cancer. Gastroenterology 2003; 125: 1626-1635

[15] Chang KJ. State of the art lecture: endoscopic ulñtrasonography (EUS) and FNA in pancreatico-biliary tumors. Endoscopy 2006; 38: 556-560

[16] Wallace MB, Pascual JM, Raimondo $M$ et al. Minimally invasive endoscopic staging of suspected lung cancer. JAMA 2008; 299: 540-546

[17] El Chafic AH, Loren D, Siddiqui A et al. Comparison of FNA and fineneedle biopsy for EUS-guided sampling of suspected gastrointestinal stromal tumors. Gastrointest Endosc 2017; 86: 510-515

[18] Iwai T, Kida M, Imaizumi H et al. Randomized crossover trial comparing EUS-guided fine-needle aspiration with EUS-guided fine-needle biopsy for gastric subepithelial tumors. Diagn Cytopathol 2018; 46: 228-233 
[19] Kim GH, Cho YK, Kim EY et al. Comparison of 22-gauge aspiration needle with 22-gauge biopsy needle in endoscopic ultrasonographyguided subepithelial tumor sampling. Scand J Gastroenterol 2014; 49: 347-354

[20] Hamada T, Yasunaga H, Nakai Y et al. Rarity of severe bleeding and perforation in endoscopic ultrasound-guided fine needle aspiration for submucosal tumors. Dig Dis Sci 2013; 58: 2634-2638

[21] Ginès A, Wiersema M], Clain JE et al. Prospective study of a Trucut needle for performing EUS-guided biopsy with EUS-guided FNA rescue. Gastrointest Endosc 2005; 62: 597-601

[22] Saftoiu A, Vilmann P, Skov BG et al. Endoscopic ultrasound (EUS)guided Trucut biopsy adds significant information to EUS-guided fine- needle aspiration in selected patiens: a prospective study. Scand J Gastroenterol 2007; 42: 117-125

[23] Wittmann J, Kocjan G, Sgouros SN et al. Endoscopic ultrasound-guided tissue sampling by combined fine needle aspiration and trucut needle biopsy: a prospective study. Cytopathology 2006; 17: 27-33

[24] Trindade AJ, Benias PC, Alshelleh M et al. Fine-needle biopsy is superior to fine-needle aspiration of suspected gastrointestinal stromal tumors: a large multicenter study. Endosc Int Open 2019; 7: E931-E936

[25] Asokkumar R, Yung KaC, Loh T. Comparison of tissue and molecular yield between fine-needle biopsy (FNB) and fine-needle aspiration (FNA): a randomized study. Endosc Int Open 2019; 7: E955-E963 